\title{
Automatic Generation of Author Output based on the Iterative Clustering Method
}

\author{
Beesung $\mathrm{Kam}^{1}$ )
}

\begin{abstract}
The structural differences and requirements of diverse techniques in searching resources, disambiguation, and bias in authors' names for bibliographic searching introduce crafting inaccuracies. On the other hand, usage of external instances of searching such as mail and venue is not sufficient for clearance in searching bibliographic data. This paper proposes iterative clustering method and labeling procedure to search a distinguished set of authors' affiliations zipped into a function to be performed on three databases having different structures. A distinguished algorithm was created using AI methodology to generate best outcomes toward reduction of bias in author's names, resulting in the enhancement of accuracy in bibliographic data. This technique also reduced the requirement of diverse manual inputs in searching resources for finding out author's national outputs. A total of 31 authors (1076 publications) were selected manually and compared against searches of three database sets RISS (Research Information System Store), KMBASE (Korean Medical Database), and DBPIA (Korea Association of Representatives) for a total of 759 found publications. $90.2 \%$ of national publication search results confirmed to be accurate.
\end{abstract}

Keywords: Author Disambiguation, Author Bias Naming, Bibliographic Generation, Labeled Data

\section{Introduction}

Although there is a rich literature on automatic bibliographic search in various databases challenges in the author name ambiguity introduce inaccuracy in crafting[1].

Searching author names in databases mostly results in the retrieval of only fraction of records. As indicated by [2] size of database might influence number of findings in resources.

Another study emphasizes that diversity in resources may increase the causal ambiguity of authors' names[3]. In this regard, an overview of the national bibliographic database of 21 nations excluding South Korea focused on the comprehensiveness of the database content compared to the content of the Web of Science and Scopus. This study reveals that despite diverse contents of databases, it is apparent that country-specific arrangements are bound to result in differences in database contents[4]. However it is not clear whether this diversity

Received(November 16, 2019), Review Result(1st: December 20, 2019, 2nd: February 04, 2020), Accepted(April 25, 2020)

1) (Professor) 50612 Dept. Medical Education, Pusan National Univ., Mul-gm-eb, Beum-au-ri, Yangsan, Korea email: beesung@pusanackr 
influences the accuracy of data retrieval.

Another study[5] suggested that contextual features, such as venue or e-mail addresses of national publications is a security-related concern and, are difficult and in some cases illegal to obtain. This article used a fraction of ambiguous author names to challenge disambiguate. However we could not afford to separate ambiguous and non-ambiguous author names. In our case distinguishing such publication documents were costly.

Supervised method train a labeled dataset and use the classifier to challenge disambiguation[6] This method is usually costly for small dataset to obtain. In the other hand unsupervised approaches, that do not need labeled data for finding similarities between nodes in publications are another approach method of clustering[7] but is limited when using complex dataset.

In relation to address ambiguity of authors' name, the Open Researcher and contributor ID (ORCID) provided persistent constant digital identifiers for author. ORCID however is an optional requirement and may not be used for national publication engines.

On the other hand the searching methods and techniques may vary across different databases. Research in [8] suggests that "search engines to produce more relevant literature" emphasizing that author name and authorship identification bias is still challenging in national publication databases.

Research at [9] shows that although the extraction of data from the database resources remains expensive, method of clusters using machine learning, decreases manual work required for bibliographic analysis. Also suggesting that by adding more detail labels a training algorithm would reduce the challenge of author name disambiguation

The objective of this study is to evaluate faculty output in which three consecutive years of previous publications can be retrieved from a national database using the author name by knowing the venue and publication year. We implemented a function to set affiliation and venue items zipped into a function to retrieve publication outputs of three national databases. We then compared our search engine data with a pre-confirmed list of publications and statistically analyzed the results.

\section{Method}

Two groups of authors were selected based on their yearly output of three consecutive years between 2016 and 2019 grouping by random and non-random frequently published authors of Pusan National University School of Medicine. This study was reviewed and exempt from 
providing consent from participants because the data analysis was conducted anonymously through public web resources and not assigning author names and output information to generate results of tables or graphs, so it did not require informed consent from participants.

A total of 31 authors (1076 publications) and their publication outputs were selected manually and separated into two groups of random and non-random frequently published authors. Each author was then nominated for searching using an AI-Based search function. Several algorithms implemented to search results from three database sets: Research Information System Store, Korean Medical Database and Korea Association of Representatives for the total of 759 found publications. By using the AI search algorithm, a list of 'confirmed publications' meaning those that were searchable within the mentioned databases, was split into two found and not found. Two other lists provided for each author named national publication outputs and international publication outputs. After searching web resources, two lists of outputs were created and compared with labeled procedure data mining. Research at [3][10-14] suggests that using e-mail as a constant key reduces bias in author name, while using a variety of different mails by authors and omitting addresses by resource providers because of privacy issues would make searching difficult to achieve[9]. Instead of this we used author affiliation and venue in addition to the iterative procedure described in [15]. The manually confirmed publications were assigned to either of the lists of publications, with manually confirmed publications and statistically analyzed compared results.

\subsection{Pre and Post-processing Data}

To search online databases, one must understand how search items should be modified to suit for posting query items in order to obtain a response suite from the web engine. Each resource requires a different technique for searching[1-3][10]. Here are some technical details that we zipped into one algorithm as a function. A hint is to prevent time consumption during the reinventing of manuals in case of re-searching items from a national database.

KBASE database uses ' $\mathrm{d}$ ' for search items and ' $\mathrm{kw}$ ' for keywords of searching requests and 'year' for publication years. Responding requests were text containing 'Doi' number, which were constant throughout the display, and we used it as a keyword to split the text for each individual publication and all other html tags were removed. Because of the none-existence of e-mail addresses[16] in this resource, we instead added sub-texts 'university' and 'medical-school' as key venue and affiliation for author search.

RISS search database engine responded to keywords like 'query', 'queryText', 'strQuery' and 
'year' of publication. Responding requests were text containing ' $<\mathrm{li}>$ ' a keyword used by html text for pending-in paragraphs and wrapped by '<em>' as an envelope. 'control_no'. 'searchViewBox' and 'University' were keywords used to split authors. All other tags were removed.

DBPIA search database responded to 'collectionQuery', 'contains', 'AUTHR' and 'AND' for the collection of mixed search options. 'ORIGINAL ARTICLES' was a keyword for searching publications because a mixture of patents, proposals, and non-registered papers was also contained in the databases.

All three databases received requests using the KSX[11] standard by adding extra values of \% between hexa-decimal values and the end. For example coding a Korean syllable '경' is mentioned as B0E6 and by adding the \% value between hexa-decimal values B0 and E6 the result will be a searchable sentence of $\mathrm{B} 0 \% \mathrm{E} 6 \%$. Returning response were in the form of 'ks_c_5601-1987' (Korean Standard enCodings). Publication dates were adjusted to three consecutive years from 2016 to 2019, and over 500 items were returned queries, for each request. Surprisingly, the process speed to post the response was less than 20s for 500 items.

\section{Results}

Two groups of authors' publications were compared against findings of an AI search algorithm. [Table 1] shows frequently out-putted published authors. In case of national publications 93.1\% of publications were found accurate, but in case of international publication search engines performed poorly at $56 \%$. Figure 1 illustrates comparison of the national publications with the AI-based search algorithm. The accuracy of finding demonstrated that AI engine worked 93\% accurate on national findings.

[Table 2] shows randomly selected authors for their publications. In the case of national publications $87.4 \%$ of publications were found accurate, while only $54.9 \%$ were accurate in the case of international publications search engine, indicating a poor performance. Figure 4 and 5 illustrate a comparison of international with national publications and AI-based search algorithm, respectively. The accuracy of finding showed that AI engine was $87.4 \%$ accurate on national findings. In total $90.2 \%$ of national publication search results were confirmed to be accurate. 
[Table 1] Frequently Outputted Authors $(\mathrm{N}=16$, total publishing output=680)

http://dx.doi.org/10.21742/apjcri.2020.05.03

International Output

$\begin{array}{cc}\text { ALL } & \text { confir } \\ \text { publi } & \text { med } \\ \text { cation } & \text { publica } \\ \text { s } & \text { tions }\end{array}$

AI

\begin{tabular}{lccccccccc} 
& $\begin{array}{c}\text { ALL } \\
\text { publi } \\
\text { cation } \\
\text { s }\end{array}$ & $\begin{array}{c}\text { confir } \\
\text { med } \\
\text { publica } \\
\text { tions }\end{array}$ & $\begin{array}{c}\text { AI } \\
\text { found } \\
\text { publi } \\
\text { cation } \\
\text { s }\end{array}$ & $\begin{array}{c}\text { AI not } \\
\text { found } \\
\text { public } \\
\text { ations }\end{array}$ & $\begin{array}{c}\text { accurac } \\
\text { (\%) }\end{array}$ & $\begin{array}{c}\text { confir } \\
\text { med } \\
\text { publica } \\
\text { tions }\end{array}$ & $\begin{array}{c}\text { AI } \\
\text { found } \\
\text { public } \\
\text { ations }\end{array}$ & $\begin{array}{c}\text { AI not } \\
\text { found } \\
\text { public } \\
\text { ations }\end{array}$ & $\begin{array}{c}\text { accurac } \\
(\%)\end{array}$ \\
\cline { 2 - 11 } auth-01 & 29 & 27 & 21 & 6 & 77.8 & 2 & 2 & 0 & 100.0 \\
auth-02 & 106 & 67 & 2 & 65 & 3.0 & 39 & 35 & 4 & 89.7 \\
auth-03 & 43 & 34 & 23 & 11 & 67.6 & 9 & 9 & 0 & 100.0 \\
auth-04 & 106 & 71 & 12 & 59 & 16.9 & 35 & 31 & 4 & 88.6 \\
auth-05 & 39 & 25 & 11 & 14 & 44.0 & 14 & 12 & 2 & 85.7 \\
auth-06 & 58 & 50 & 36 & 14 & 72.0 & 8 & 8 & 0 & 100.0 \\
auth-07 & 41 & 40 & 35 & 5 & 87.5 & 1 & 1 & 0 & 100.0 \\
auth-08 & 74 & 54 & 13 & 41 & 24.1 & 20 & 18 & 2 & 90.0 \\
auth-09 & 24 & 21 & 15 & 6 & 71.4 & 3 & 3 & 0 & 100.0 \\
auth-10 & 12 & 11 & 7 & 4 & 63.6 & 1 & 1 & 0 & 100.0 \\
auth-11 & 18 & 17 & 14 & 3 & 82.4 & 1 & 1 & 0 & 100.0 \\
auth-12 & 29 & 15 & -2 & 17 & -13.3 & 14 & 12 & 2 & 85.7 \\
auth-13 & 26 & 24 & 17 & 7 & 70.8 & 2 & 1 & 1 & 50.0 \\
auth-14 & 28 & 28 & 20 & 8 & 71.4 & 0 & 0 & 0 & 100.0 \\
auth-15 & 14 & 13 & 11 & 2 & 84.6 & 1 & 1 & 0 & 100.0 \\
auth-16 & 33 & 33 & 24 & 9 & 72.7 & 0 & 0 & 0 & 100.0 \\
\hline
\end{tabular}

[Table 2] Randomly Selected Authors ( $N=15$, total publishing output=396)
National Output

\begin{tabular}{ccccccccc}
$\begin{array}{c}\text { ALL } \\
\text { publi } \\
\text { cation } \\
\text { s }\end{array}$ & $\begin{array}{c}\text { confir } \\
\text { med } \\
\text { publica } \\
\text { tions }\end{array}$ & $\begin{array}{c}\text { AI } \\
\text { found } \\
\text { publi } \\
\text { cation } \\
\text { s }\end{array}$ & $\begin{array}{c}\text { AI not } \\
\text { found } \\
\text { public } \\
\text { ations }\end{array}$ & $\begin{array}{c}\text { accurac } \\
(\%)\end{array}$ & $\begin{array}{c}\text { confir } \\
\text { med } \\
\text { publica } \\
\text { tions }\end{array}$ & $\begin{array}{c}\text { AI } \\
\text { found } \\
\text { public } \\
\text { ations }\end{array}$ & $\begin{array}{c}\text { AI not } \\
\text { found } \\
\text { public } \\
\text { ations }\end{array}$ & $\begin{array}{c}\text { accurac } \\
(\%)\end{array}$ \\
\cline { 2 - 8 } 10 & 27 & 21 & 7 & 77.8 & 6 & 6 & 0 & 100.0 \\
19 & 67 & 2 & 2 & 3.0 & 1 & 1 & 0 & 100.0 \\
23 & 74 & 23 & 2 & 67.6 & 4 & 2 & 2 & 50.0 \\
99 & 25 & 12 & 14 & 16.9 & 14 & 13 & 1 & 92.9 \\
32 & 50 & 36 & 3 & 72.0 & 0 & 0 & 0 & 100.0 \\
4 & 40 & 35 & 4 & 87.5 & 4 & 4 & 0 & 100.0 \\
19 & 54 & 13 & 2 & 24.1 & 3 & 1 & 2 & 33.3 \\
35 & 21 & 15 & 23 & 71.4 & 24 & 21 & 3 & 87.5 \\
8 & 11 & 7 & 3 & 63.6 & 3 & 2 & 1 & 66.7 \\
25 & 17 & 14 & 10 & 82.4 & 9 & 9 & 0 & 100.0 \\
16 & 15 & -2 & 1 & -13.3 & 1 & 1 & 0 & 100.0 \\
10 & 24 & 17 & 5 & 70.8 & 3 & 3 & 0 & 100.0 \\
51 & 28 & 20 & 21 & 71.4 & 20 & 20 & 0 & 100.0 \\
24 & 13 & 11 & 16 & 84.6 & 18 & 16 & 2 & 88.9 \\
396 & & 235 & & 54.9 & & 130 & & 87.4
\end{tabular}


[Table 3] Differentiating Non-rand and Random Groups and Publication Output (N=31) International Output

National Output

\begin{tabular}{|c|c|c|c|c|c|c|c|c|c|}
\hline & $\begin{array}{c}\text { ALL } \\
\text { publi } \\
\text { cation } \\
\text { s }\end{array}$ & $\begin{array}{c}\text { confir } \\
\text { med } \\
\text { publica } \\
\text { tions }\end{array}$ & $\begin{array}{c}\text { AI } \\
\text { found } \\
\text { publi } \\
\text { cation } \\
\mathrm{s} \\
\end{array}$ & $\begin{array}{l}\text { AI not } \\
\text { found } \\
\text { public } \\
\text { ations }\end{array}$ & $\begin{array}{c}\text { accurac } \\
\mathrm{y} \\
(\%)\end{array}$ & $\begin{array}{c}\text { confir } \\
\text { med } \\
\text { publica } \\
\text { tions }\end{array}$ & $\begin{array}{c}\text { AI } \\
\text { found } \\
\text { public } \\
\text { ations }\end{array}$ & $\begin{array}{l}\text { AI not } \\
\text { found } \\
\text { public } \\
\text { ations }\end{array}$ & $\begin{array}{c}\text { accurac } \\
\mathrm{y} \\
(\%)\end{array}$ \\
\hline \multirow{2}{*}{ 31Authors } & 680 & 530 & 259 & 271 & 56.0 & 150 & 135 & 15 & 93.1 \\
\hline & 396 & 497 & 235 & 173 & 54.9 & 144 & 130 & 14 & 87.4 \\
\hline
\end{tabular}

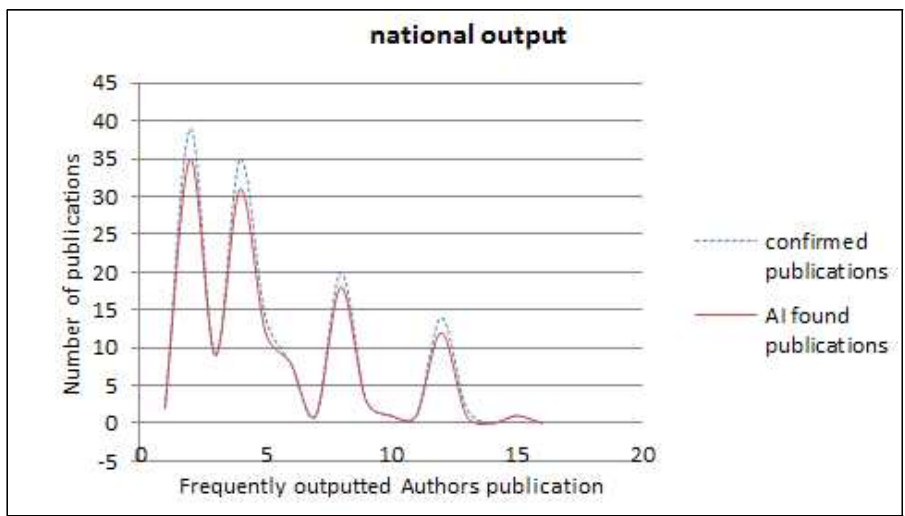

[Fig. 1] Illustration of National Publications Against Findings of AI-Based Algorithm in case of Frequently Outputted Authors

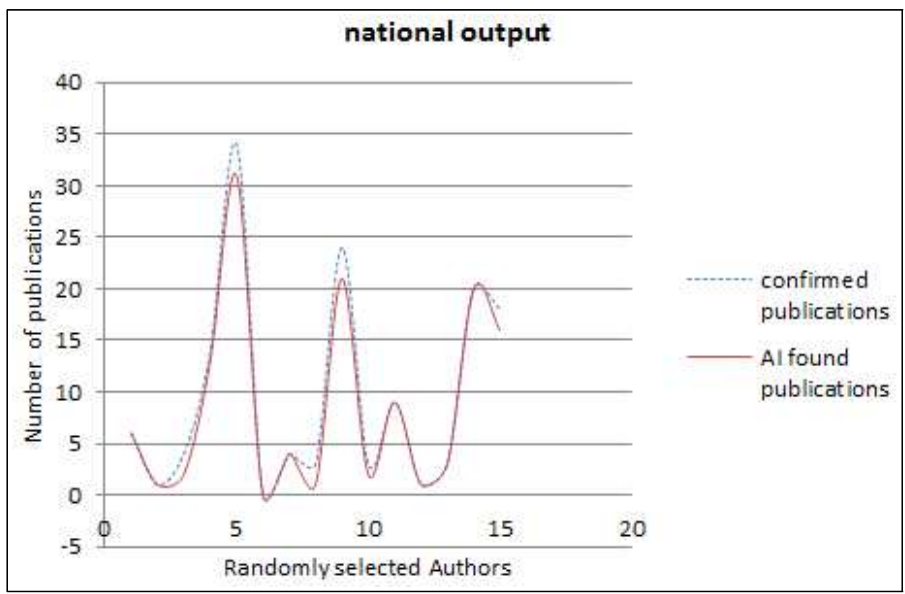

[Fig. 2] Illustration of National Publications Against Findings of AI-Based Algorithm in the case of Randomly Selected Authors 


\section{Conclusion}

To address the ambiguities associated with author names in resources to enhance retrieval of publications by searching the name of authors, we developed and implemented name labeling technique on three well-known search engines.

In [9] the authors proposed that lack of information on search queries can result in disambiguation of names the author names. From this study, introducing constant labelling by using a machine learning technique to form clusters for searches by names of authors yielded $76.4 \%$ accuracy. However, the limitation of this study is the inability to obtain a complete list of publication author names. This research is inspired by the requirement of validating results via external sources.

A graph topological feature was introduced in [6] to address name ambiguities without privacy-related problems. The input for this method was based on ambiguous author names and their relation with the similarity of nodes based on neighbor structures. The study considered only ambiguous publication outputs. Their method used person-to-person collaborations by introducing person to document and document-to-document networks to enhance the accuracy of the outputs. However, we considered all publication outputs including ambiguous and unambiguous.

A logistic regression and hierarchical agglomerative clustering was introduced in [17] to learn from supervised information. The study used a single fixed external threshold, including publication title, coauthor, venue, and year. However, the limitation of this study was the cost of performance. In contrast, although this study introduced "Title" as an external resource required for retrieval, it is a component of the object of study.

A study[15] closely related to our study proposed an automatic labeling method that integrates ORCID IDs, name instances, mail address, self-citation and co-authorship, and it yields maximum of $99.91 \%$ accuracy in bibliographic search data. Owing to the alternative development of mail addresses, a large number of spam and mailing category[2], mail addresses can not be an accurate tool to clarify ambiguous author's name. To address this issue we used venue, school name, and major specified in each publication.

In this study, we focused on training a similar algorithm and introducing additional options for using different labeling procedures. The solutions mentioned above are based on external sources of biographical attributes retrieved from pre-publishing documents. However, although this does not use an external authority database, it relies on online national databases. 
However, the limitation of this study is the use of a limited number of database engines. In future works we plan to extend our search to internationally focused database engines. Furthermore, this study did not retrieve all publication outputs after international and national publications are introduced. The fraction of international output identified from the national database was presented on the left side of [Table 1],[Table 2], and [Table 3], representing 55\% accuracy after validating the output. Thus, an extra function to aid retrieving international output by integrating with the current function must be explored. However, this is a work in progress, that can enhance the functional usability of this study in the future. Despite understanding the influence of diversity of resources on the accuracy of data retrieval, focus can be extended to address this question in later publications.

\section{Acknowledgement}

This work was supported by medical research institute grant from Pusan National University School of Medicine (planning assignment), in 2019.

\section{References}

[1] N. R. Smalheiser, A. M. Cohen, Design of a generic, open platform for machine learning-assisted indexing and clustering of articles in PubMed, a biomedical bibliographic database, Data and Information Management, (2018), Vol.2, No.1, pp.27-36. DOI: 10.2478/dim-2018-0004

[2] W.B. Croft, D. Metzler, T. Strohman, Search engines: Information retrieval in practice, Pearson Education, (2015)

[3] J. S. Kim, Evaluating author name disambiguation for digital libraries: a case of DBLP, Scientometrics, (2018), Vol.116, No.3, pp.1867-1886. DOI: 10.1007/s11192-018-2824-5

[4] L. Sīle, J. Pölönen, G. Sivertsen, R. Guns, T. C. E. Engels, P. Arefiev, M. Duškova, L. Faurbaek, A. Holl, E. Kulczycki, B. Macan, G. Nelhans, M. Petr, M. Pisk, S. Shoos, J. Stojanovski, A. Stone, J. Šušol, R. Teitelbaum, Comprehensiveness of national bibliographic databases for social sciences and humanities: Findings from a European survey, Research Evaluation, (2018), Vol.27, No.4, pp.310-322. DOI: 10.1093/reseval/rvy016

[5] S. Konlechner, V. Ambrosini, Issues and Trends in Causal Ambiguity Research: A Review and Assessment, Journal of Diversity, (2019), Vol.45, No.6, pp.2352-2386.

[6] B. Zang, M.A. Hasan, Name Disambiguation in Anonymized Graphs using Network Embedding, Proceedings of the 2017 ACM on Conference on Information and Knowledge Management, (2017), November 6-10; Singapore, DOI: https://doi.org/10.1145/3132847.3132873 
[7] Shen Q, Wu T, Yang H, Wu Y, Qu H, Cui W, NameClarifier: A Visual Analytics System for Author name Disambiguation, IEEE Trans Vis Comput Graph, (2016), Vol.23, No.1, pp.141-150. DOI: 10.1109/TVCG.2016.2598465

[8] C. Stansfield, K. Dickson and M. Bangpan, Exploring issues in the conduct of website searching and other online sources for systematic reviews: how can we be systematic?, Systematic Reviews, (2016), Vol.5, No.1, pp.191. DOI: $10.1186 / \mathrm{s} 13643-016-0371-9$

[9] M. A. Abdulhayoglu, B. Thijs, Use of ResearchGate and Google CSE for author name disambiguation, Scientometrics, (2017), Vol.111, No.3, pp.1965-1985. DOI: 10.1007/s11192-017-2341-y

[10] M. C. Muller, F. Reitz, N. Roy, Data sets for author name disambiguation: an empirical analysis and a new resource, Scientometrics, (2017), Vol.111, No.3, pp.1467-1500. DOI: 10.1007/s11192-017-2363-5

[11] http://kbox.kaist.ac.kr/resource/KS_X_1001/accessed, Sep 16 (2019)

[12] S. Byun, Design of Efficient Index Management for Column-based Big Databases, International Journal of Internet of Things and Big Data, (2017), Vol.2, No.1, pp.59-64. DOI: 10.21742/ijitbd.2017.2.1.06

[13] H. Singh, D. Tomar, S. Agarwal, Link Prediction for Authorship Association in Heterogeneous Network Using Streaming Classification, International Journal of Grid and Distributed Computing, (2016), Vol.9, No.4, pp. 135-150. DOI: 10.14257/ijgdc.2016.9.4.13

[14] S. Saxena, G. Sanyal and S. Srivastava, Enhancement of Cloud Authorization System Using Predicate Logic, International Journal of Security and Its Applications, (2017), Vol.11, No.1, pp.245-256. DOI: 10.14257/ijsia.2017.11.1.19

[15] L. Cen, E. C. Dragut, L. Si, M.. Quzzani, Author disambiguation by hierarchical agglomerative clustering with adaptive stopping criterion, The $36^{\text {th }}$ International ACM SIGIR Conference on Research and Development in Information Retrieval, (2013), July 28-August 1; Dublin, Ireland.

[16] J. S. Kim, J. Kim, The impact of imbalanced training data on machine learning for author name disambiguation, Scientometrics, (2018), Vol.117, No.1, pp.511-526. DOI: 10.1007/s11192-018-2865-9

[17] J. S. Kim, J. Kim. J. O. Smith, Generating automatically labeled data for author name disambiguation: an iterative clustering method, Scientometrics, (2019), Vol.118, No.1, pp.253-280. DOI: 10.1007/s11192-018-2968-3 